\title{
Design Principles of External Lighting for Architectural Objects
}

\author{
Batova Anastasia \\ Moscow Architectural Institute (State Academy), Moscow, Russian Federation
}

\begin{abstract}
Nowadays, lighting design is the modern attractive field of professional activity. Although its theoretical and methodological bases are in the initial stage of formulation. Though there are standards for regulating illumination quantitatively, the science has never created a bridge to transfer illumination quantity into illumination quality. How to keep a day image of architectural object without imitating natural lighting? How unrecognizably to change the image, to make it extraordinary and catchy? This paper offers a solution in the form of external illumination designing principles. This paper proposes two conceptual ways of creating artificial lighting of buildings and constructions that pursue different goals: the principle of "tectonic" illumination and the principle of "decorative" illumination. These principles were developed based on the analysis of the state of the art experience of lighting architectural objects and author's empirical study. The latter contained measuring luminosity with luminance meter, processing the data, statistical survey (180 respondents) and lighting modeling in the laboratory of Moscow Architectural Institute.
\end{abstract}

Keywords: lighting design, architecture, principle, tectonics, image

\section{Introduction}

This paper is devoted to the problem of creating a predicted image of building by artificial light. Illumination is as much a "constructional material" of creating similitude as stone, wood, metal, glass, and concrete. Construction material as a component of the whole building construction in many respects dictates the architectural image. In the same way, light historically designates a problem of figurative perception dependence on solar or cloudy lighting in the daytime and artificial light at night.

This paper aims to zoom in external illumination design principles generated by the author. There is the Principle of "Decorative" Lighting and the Principle of "Tectonic" Lighting. The former is directed on creation a new original architectural image without natural archetype, and the latter is focused on transfer of initial (day) emotional impression into the nighttime, without imitating day light. Pluses and minuses, means and methods of creation are described in the paper. These principles are formulated first on the basis of the analysis and systematization of external illumination world experience, and second on the author's researches including on-site investigations, statistical (actuarial) poll, and laboratory light modeling.

\section{Benchmark}

The art of the external illumination of architectural objects and ensembles has been actively developed during the 20th century and got the independence status nowadays. However, in practice intuitive, empirical approach is still dominating.

Batova Anastasia, Ph.D., Department of Architectural Physics, Moscow Architectural Institute (State Academy). 
Lighting is the main component of visual impression. Image similarity formed by day (natural) lighting and by night-time (artificial) lighting is defined by similarity of experience consistency of impression. Certainly, recognition occurs in consequence of configuration, but it is not limited to that. No less importance is laid on emotions, feelings, and associations being evoked by the illuminated object. According to the aforesaid, the object's image means implies its sensual and evident perception.

The research is based on the concept "tectonics" as a system of architectural forms classification. Tectonics means "art expression of behavior of structure and construction material, entailing specify object perception by viewer, these are mechanically stable and robust... The tectonics of construction comes out of design and behavior of a material and inseparable from them" (Ikonnikov, 1971, p. 65). Therefore, analysis of tectonic systems is normally connected with main types of designs: wall, order, arch, frame system, etc.. So, for example, for wall tectonics visual solidity, continuity of wall plays an important role apart from visual stability and durability, for order systems there appears communication of a colonnade (rack) and an entablature (beam), visual perception of portico or gallery depth.

The research brought forward two principles of external illumination designing: the Principle of "Decorative" Lighting and the Principle of "Tectonic" Lighting, which differ in purposes, tasks, and methods to achieve.

\section{Principle of "Decorative” Lighting}

Decorative lighting gets the aim to create a new original image which does not have a natural archetype. It is created by regular or irregular (picturesque) brightness distribution on architectural object surfaces. Usually, it is characterized by high brightness contrast (L1:L2 $\geq 1: 10)^{1}$ with sharp, non-uniform light-and-shade thresholds. Regular distribution of brightness as a rule tends to be in phase with an arrangement of plastic elements. High brightness contrast visually making dominant secondary elements, thus entails brings forward a new system of relationship between major and minor architectural elements defining a new object image, significantly different from a day archetype. For example, bright illuminated semi-columns on the unlit surface of a wall tectonics facade create the light rhythm visually crashing solidity of a wall and highlighting making the order tectonics dominant. Irregular distribution of brightness completely ignores architectural surface plastic elements creating new "picture", for instance, by video mapping (see Figure 1).
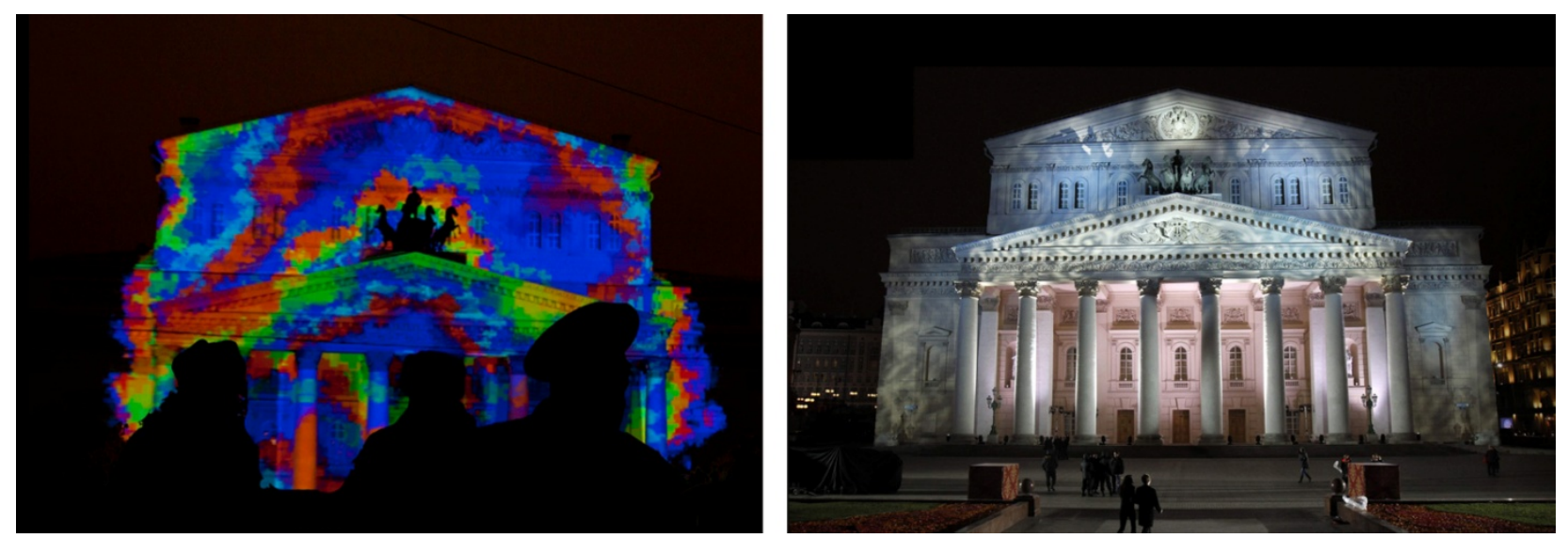

Figure 1. Illumination of the Bolshoy Theater facade.

\footnotetext{
${ }^{1}$ Here: Brightness - the average brightness of an object (e.g., a column), background brightness — the average brightness of a background the object is projected (e.g., a wall).
} 
Unique features of the images created by the Principle of "Decorative" Lighting are stylistic uniformity and visual flatness. This image (being created on high brightness and color contrasts) thrills the viewer, being grasped in a single flash, still it prevails over its day-time archetype. As a result, the reapplied lighting composition scheme creates an identical image of any other object (see Figures 2-3). Failed examples of decorative lighting split the object up in an illusory way tending to provoke the effect of internal in the observer.

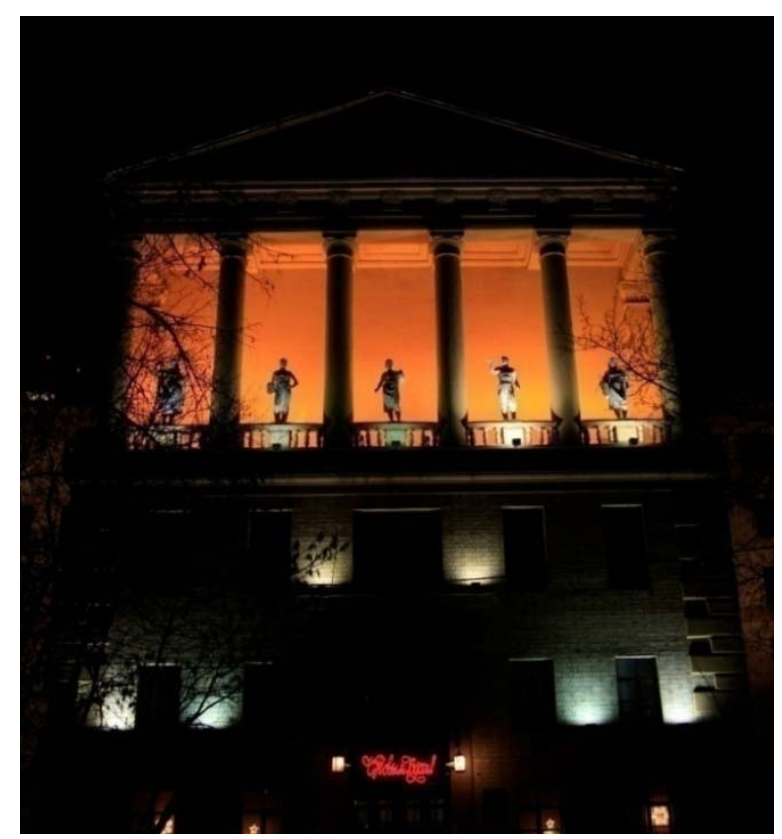

Figure 2. Illumination of the Stroganovskaya Akademy fasade.

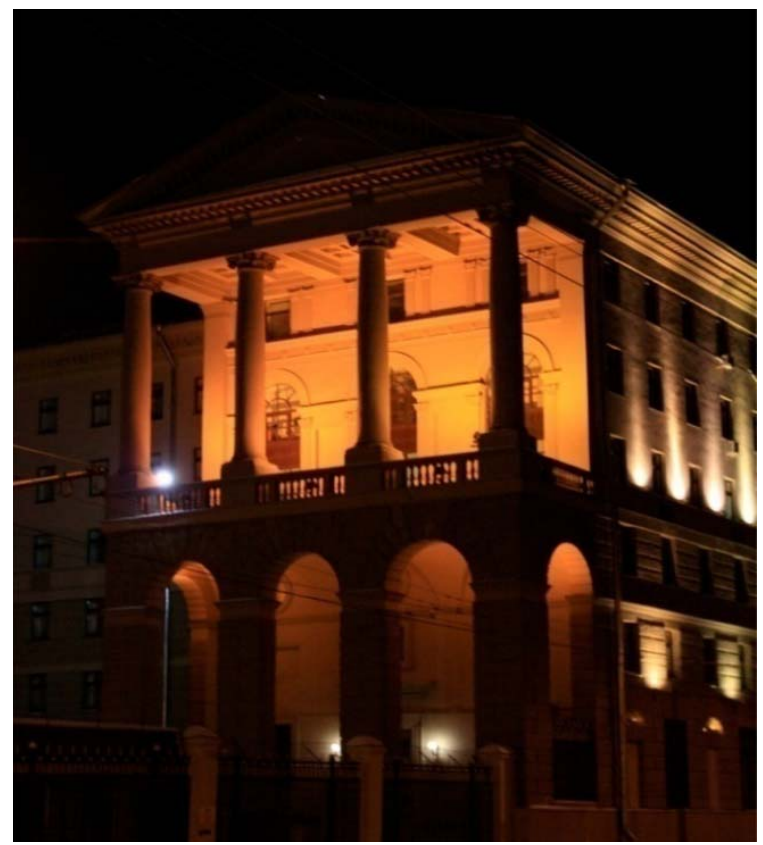

Figure 3. Illumination of the fasade of the building (Petrovka st., 38).

The Principle of "Decorative" Lighting, in condition of developing an individual, non-duplicated lighting scheme for the given architectural object should becomes a base of lighting design masterpieces. 


\section{Principle of "Tectonic" Lighting}

The Principle of "Tectonic" Lighting aims at visual detection of tectonic features of the object, directed not only on visual recognition of the object, but also on the most effective figurative interpretation of the composite decision, on transfer of initial (day) emotional impression which is mostly predetermined by its tectonics. Its applicability in lighting historical architecture gets especially topical for illumination monuments of historical and cultural heritage which have the right to be recognizable any time, i.e., to protect constancy of the unique architectural artistic image.

This principle of lighting assumes using specific lighting schemes of brightness distribution on designated elements of architectural composition. In other words, the lighting composition is always rigidly attached to the given architectural elements defining a tectonic image of selected system.

It was experimentally proved that each of the tectonic types explored demands individual recommendations for the brightness contrast on the key elements of an architectural composition (i.e., it is incorrect to apply the same illumination schemes for different tectonic architecture in types). So, for wall tectonics objects, the key feature is the brightness contrast in illuminated and unlit zones of the plane, while for order tectonics and arch and vaulted architecture the contrast within certain elements of the fore and middle ground is dominant ${ }^{2}$. The research proved that exactly balance of average brightness had played a crucial role in perception of architecture at night-time instead of their quantitative levels; brightness of adaptation is of secondary importance. Thus, the searched ratio should be recommended for whatever object's location in city, be it a central square full of bright light or a secluded dark lane. Putting it differently, in the "tectonic" way of lighting unlike the "decorative" one reapplied lighting schemes do not lose their relevance. Because each time they reveal constructive, art and figurative features of various architectural object, working for creating a new original image.

Lighting schemes being searching out for wall, order, arch and vaulted tectonic systems and being translated into the language of illumination engineering terms as a brightness correlation, are nominally called "Over contrast", "Normal contrast", and "Scarce contrast".

The field of the Principle of "Tectonic" lighting potential application is extremely extensive, and is never limited by objects with a certain and obviously expressed tectonics. "Architecture can be diverse in manifestations. Each era displays specific receptions of art judgment of a design" (Ikonnikov, 1971, p. 82). Tectonic forms strictly specified by structure at the beginning, in the subsequent development got an opportunity to be harmoniously and equally combined in a uniform composition of an architectural object. There examples are plentiful: the Pantheon in Rome, monument of antique architecture, uniting equivalent from the composite point of view an order portico and the dome arch, the Palace of rains in Venice, combining tectonics of a wall and an arch and vaulted design, the building of Smolny Institute in St. Petersburg, here adjoin order and wall tectonic systems. Also there exists a considerable group of buildings and the constructions where architectural form expresses behavior of structure not obviously but indirectly. So, for instance, the order being created by antiquity as tectonic system later came to be applied as the decorative element deprived of borne and bearing functions.

The issue of developing sophisticated architectural forms combining several constructive artistically interpreted systems by artificial light, is one of the great core intellectually tasks for a lighting designer.

\footnotetext{
${ }^{2}$ E.g., tectonics of order architecture defines a ratio of average brightness of a colonnade with an entablature (L1) and a background wall (L2). "Normal contrast" is L1:L2 = 1:3.
} 
Depending on task at hand the hierarchy of tectonic systems should be kept (following "reference" day image) or be broken in the dark. According to the Principle of "Tectonic" Lighting, the system illuminated in the terms of "Normal" brightness contrast should become visually prevalent, subordinated by the one illuminated in terms of "Scarce contrast". The above described provisions are being demonstrated by the visualizations of the Bolshoy Theater facade lightning, as an example of an object combining order and wall tectonic systems.

The first case (see Figure 4(a)) presents the lighting scheme where the order portico lighted more contrasty and accurately forms the whole object's image. The next case visually putting the wall in the forefront and making the wall system leading (see Figure 4(b)). Depth of architectural composition is visually lost, the general silhouette and mass of the building dematerialized to a certain extent by bright light, on the contrary, gain the central focus. The third case presents visualization where not any of tectonic systems is unambiguously preferred (see Figure 5). The visual interrelation based on the planned character of the general architectural composition of the facade comes to light. The final cast is turns the most complicated from the art point of view, however, it helps to create the most interesting and attractive light images in the night city landscape.

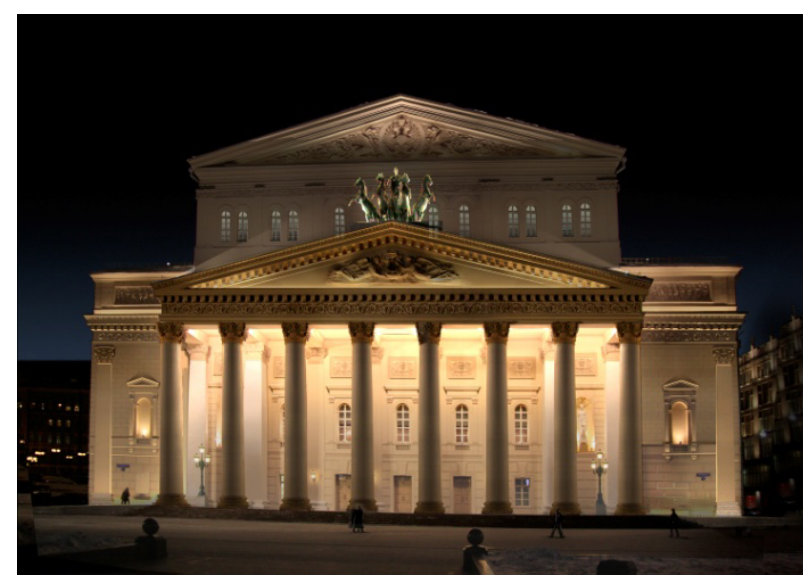

(a)

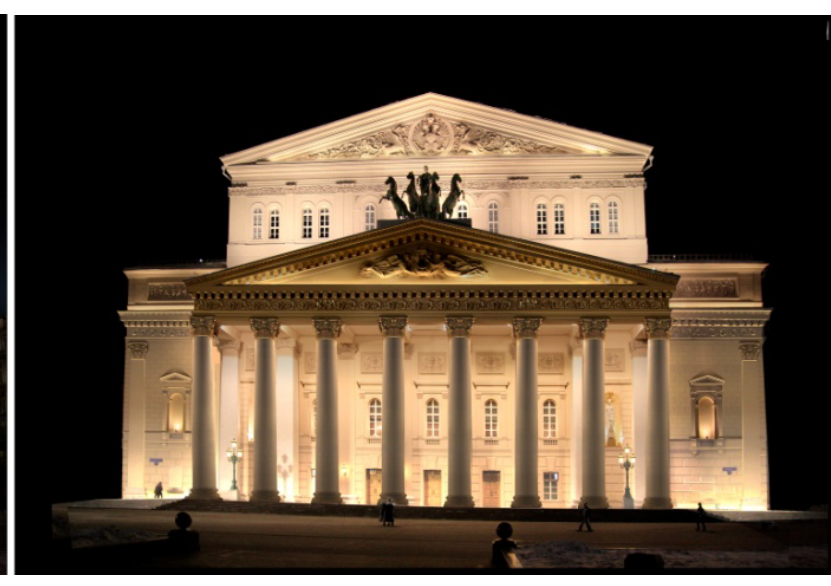

(b)

Figure 4. Illumination of the Bolshoy Theater facade (project).

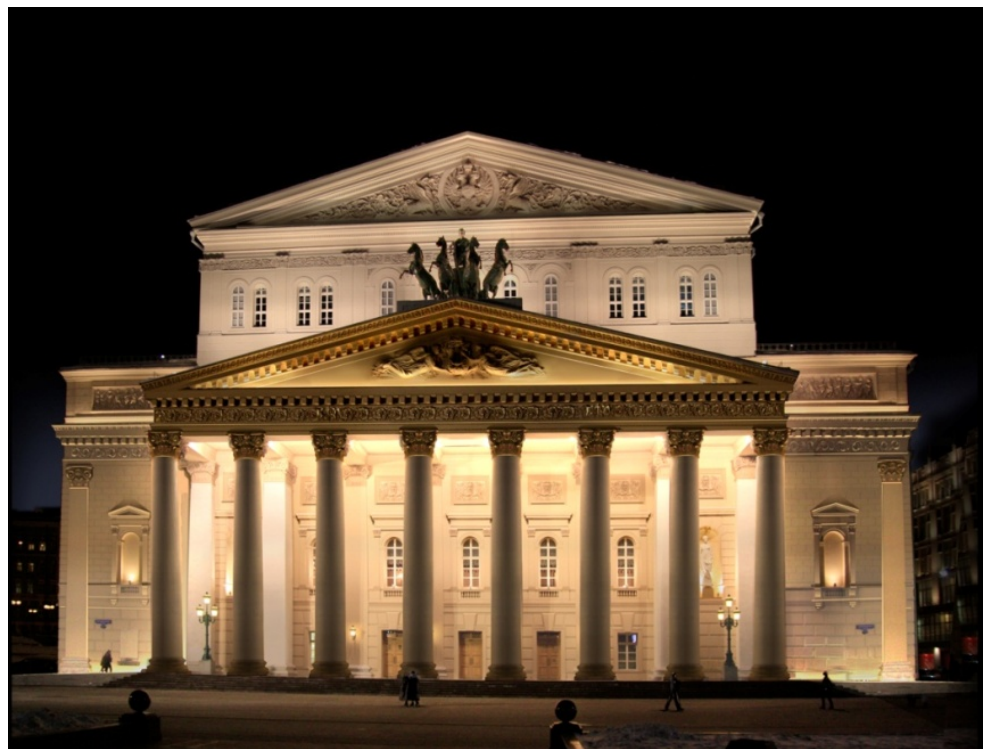

Figure 5. Illumination of the Bolshoy Theater facade (project). 


\section{Conclusions}

\section{Objective}

The Principle of "Decorative" Lighting: to create a new, original night image of an illuminated object (a new illusory form, new impression) comparative day archetype;

The Principle of "Tectonic" Lighting: to create a new illusory form of illuminated object, preserve related still preserve it closely related to a day archetype impression.

\section{Methods to Achieve}

The Principle of "Decorative" Lighting: distribution of brightness is not attached to an architectural form. As a rule the brightness contrast is strong (L1:L2 $\geq 1: 10$ ), light and shade thresholds are sharp, non-uniform.

The Principle of "Tectonic" Lighting: distribution of brightness is rigidly attached to key elements of architectural form. The Key elements are governed by tectonic type. As a rule the brightness contrast is normal (average) (1:2 $\leq \mathrm{L} 1: \mathrm{L} 2 \geq 1: 8)$, light and shade thresholds are soft.

\section{Specific Application Features}

The Principle of "Decorative" Lighting: lighting scheme duplication entails image-cloning. The new object calls for originating a new scheme.

The Principle of "Tectonic" Lighting: lighting scheme duplication entails a great variety of images dedicating identity for each of the illuminated objects. The lighting scheme investigated for wall tectonic should be used as much as you like for any wall tectonic building or structure to be origin; lighting scheme investigated for order tectonic should be used as much as you like for any order tectonic building or structure to be origin, etc.

It is mostly plausible in illuminating monuments of historical and cultural heritage.

\section{References}

Azizyan, I. A. (2002). Composition theory as architecture poetics (Teoriya kompozicii kak poehtika arkhitekturih) (p. 476). I. A. Azizyan, I. A. Dobricihna, \& G. S. Lebedeva (Eds.). Moscow: Progress-Traditions (Progress-Tradicii).

Danler, A. (2011). Building with natural light-A symbiosis of light, space and sustainability (pp. 141-144). Gütersloh: Via-Verlag.

Iannone, F. (2011). Ascona: Night light sets made by natural daylight (pp. 103-105). Gütersloh: Via-Verlag.

Ikonnikov, A. V. (1971). Bases of architectural composition (Osnovih arkhitekturnoyj kompozicii). Moscow: Stroiizdat.

Karcher, A., Krautter, M., Kuntzsch, D., Schielke, T., Steinke, C., \& Takagi, M. (2009). Light perspectives, between culture and technology: Light, space, perspectives. Lèudenscheid: ERCO.

Keller, M. (2010). Light fantastic: The art and design of stage lighting (3rd ed.). Munich: Prestel.

Laganier, V., \& van der Pol, J. (Eds.). (2011). Light and emotions: Exploring lighting cultures: Conversations with lighting designers (2nd ed.). Basel: Birkhäuser.

Neumann, D. (2002). Architecture of the night: The illuminated building. Munich and New York: Prestel. 